\title{
in silico Exploration of Repurposing and Optimizing Traditional Chinese Medicine Rutin for Possibly Inhibiting SARS-CoV-2's \\ Main Protease
}

\author{
Tien Huynh, ${ }^{\dagger}$ Haoran Wang, ${ }^{\ddagger}$ Wendy Cornell, ${ }^{\dagger}$ and Binquan Luan ${ }^{*}, \dagger$ \\ $\dagger$ †omputational Biological Center, IBM Thomas J. Watson Research, Yorktown Heights, \\ New York 10598, USA \\ $\ddagger$ Neoland Biosciences, Medford, Massachusetts, 02155, USA \\ E-mail: bluan@us.ibm.com
}

\begin{abstract}
Coronavirus disease 2019 (COVID-19) is an ongoing global pandemic with very limited specific treatments. To fight COVID-19, various traditional antiviral medicines have been prescribed in China to infected patients with mild to moderate symptoms and received unexpected success in controlling the disease. However, the molecular mechanisms of how these herbal medicines interact with the virus have remained elusive. It is well known that the main protease (Mpro) of SARS-CoV-2 plays an important role in maturation of many viral proteins such as the RNA-dependent RNA polymerase. Here, we explore the underlying molecular mechanisms of the computationally determined top candidate-rutin, a key component in many traditional antiviral medicines such as Lianhuaqinwen and Shuanghuanlian, for inhibiting the viral target-Mpro. Using in silico methods (docking and molecular dynamics simulations), we revealed the dynamics and
\end{abstract}


energetics of rutin when interacting with the Mpro of SARS-CoV-2, suggesting that the highly hydrophilic rutin molecule can be bound inside the Mpro' pocket (active site) and possibly inhibit its biological functions. In addition, we optimized the structure of rutin and designed a more hydrophobic analog which satisfies the rule of five for western medicines and demonstrated that it possesses a much stronger binding affinity to the SARS-COV-2's Mpro.

The ongoing pandemic of respiratory disease spreading from person-to-person is caused by a novel coronavirus named novel severe acute respiratory syndrome coronavirus 2 (SARSCoV-2). The disease itself has been officially called coronavirus disease 2019 (COVID-19) as it first emerged at the end of 2019. Coronaviruses are common in humans and different species of animals such as birds, bats, pigs and camels. In general, animal coronaviruses rarely infect people except the ones that cause zoonotic diseases like this newly identified SARS-CoV-2 as well as the severe acute respiratory syndrome coronavirus (SARS-CoV) ${ }^{1}$ and Middle East respiratory syndrome coronavirus (MERS-CoV) ${ }^{2}$ that have resulted in largescale outbreaks not too long ago. Although COVID-19 appears less deadly than both SARS and MERS, it is highly contagious and has touched almost every corner of the globe, infecting more than 4 million people in just a few months. Its economic consequences are impacting the whole world with unprecedented speed and severity due to the fact that many countries have to impose lockdowns and close borders to contain the spread of the virus because, to date, there is no efficient and specific antiviral treatment for COVID-19 unfortunately.

Despite majority of COVID-19 patients experience only mild illness and are able to recover without medical care, the ones with serious underlying medical conditions could get severely sick with life-threatening complications of pneumonia that require hospitalization. Various promising drug molecules developed for treating other viral infections (such as Remdesivir) are currently under clinical trials. In China, the traditional herbal antiviral medicine such as Lianhuaqingwen (LHQW) has been used to treat COVID-19 patients and demonstrated the outstanding control of the disease. Experimentally, herbal medicines in- 
cluding LHQW was found to significantly inhibit the replication of SARS-CoV-2 in vitro, ${ }^{3}$ but the mechanism is still not well understood.

As a major component of many herbal antiviral medicines, rutin and its analogs had been reported to inhibit a broad spectrum of proteases, such as the viral Mpro (3CL protease) of SARS-CoV ${ }^{4}$ enteroviruses (EV71), ${ }^{5} \mathrm{HuNoV}$ proteases, ${ }^{6}$ or even human thrombin protease. ${ }^{7}$ Besides, rutin has also been found to inhibit the multiplication of parasites, bacteria, fungi and viruses. ${ }^{8}$ Recently, rutin was also reported to be a top hit for protein disulfide isomeras (PDI) inhibitor, potentially explaining its potent anti-coagulation activity, a critical beneficial feature in the treatment of COVID-19 patients. ${ }^{9}$ In fact, Troxerutin, a commonly used rutin analog, was often prophylactically prescribed among COVID-19 patients to prevent blood coagulation and protect the cardiovascular system. Recently, LHQW had been widely used for treating COVID-19 patients and it was proved to inhibit SARS-CoV-2 virus in vitro. ${ }^{10}$ Indeed, rutin can be found in the bloodstream after the intake of LHQW. ${ }^{11}$

Currently, it is well known that the SARS-CoV-2's main protease (Mpro) constitutes one of the most attractive antiviral drug targets, because the viral protein maturation relies heavily on the viral Mpro's activities. The twelve viral non-structural proteins (Nsp4-Nsp16), including critical proteins like the RNA-dependent RNA polymerase (RdRp, Nsp12) and helicase (Nsp13), require the cleavage through the Mpro. It has also been demonstrated experimentally that inhibition of the Mpro prevented viral replication in multiple cell models with COVID-19 pseudovirus as well as SARS2-CoV-2 virus. ${ }^{12}$ Virus enters cell via different proteases and receptors in different organs. Efficacy of viral entry inhibitors like Chloroquine/Hydroxychloroquine may be highly dependent on the organ receptor/protease expression profile. ${ }^{13}$ However, Mpro activities are required for virus proliferation in all cell types, and thus warrant further research and development efforts with a high-priority. In this work, we are motivated to investigate the possibility of Mpro's inhibition by rutin.

It is essential to gain insights into the molecular mechanisms behind the binding of a ligand to its protein target in order to design safe and effective drugs with high specificity 


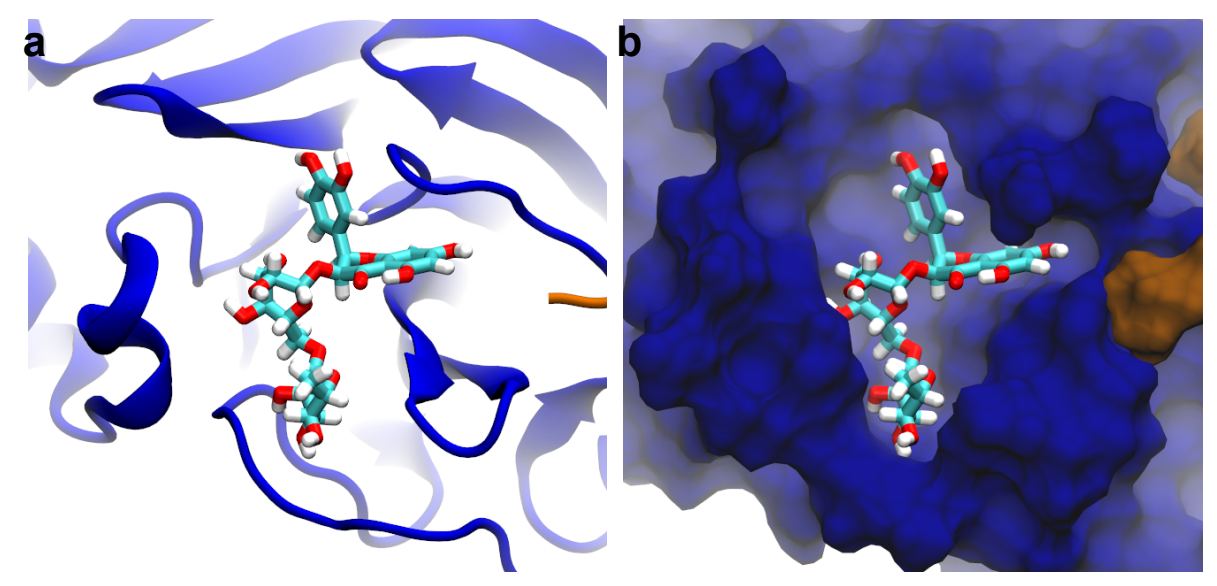

C

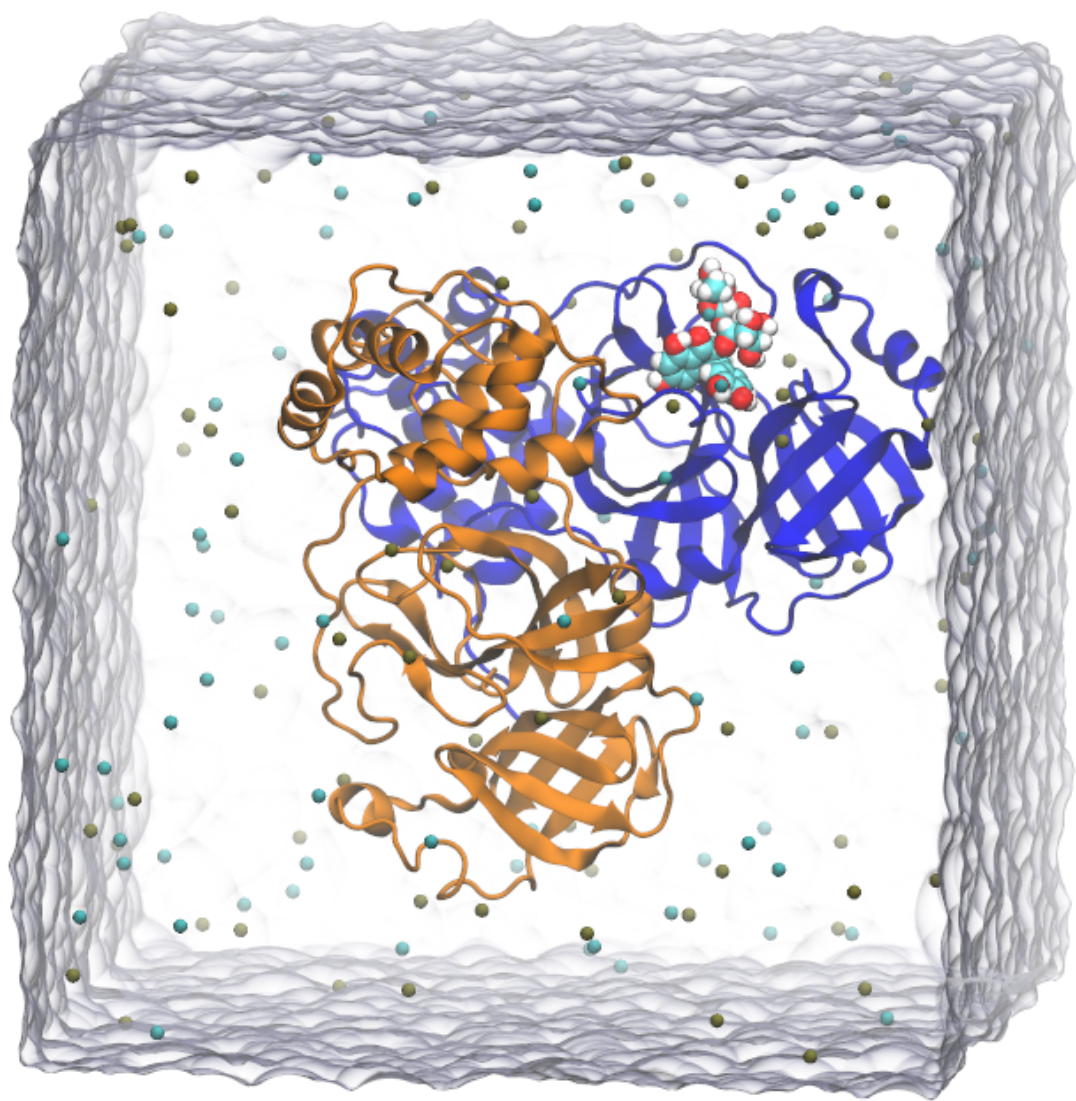

Figure 1: The simulation models for rutin bound inside the Mpro's pocket. a,b) the top pose of rutin inside the Mpro's pocket from the docking study. The rutin molecule is in the stick representation. The rutin-bound monomer (blue) is in the cartoon representation (a) and in the molecular surface representation (b). The N-terminal of the other monomer that is near the rutin's binding site is shown in orange. c) An illustration of the molecular dynamics system. Two monomers (colored in orange and blue respectively) in the Mpro dimer are in cartoon representation. $\mathrm{K}^{+}(\tan )$ and $\mathrm{Cl}^{-}$(cyan) ions are shown as van der Waals spheres. Water is shown transparently. 
and affinity for treatment of the underlying disease. Here, we employed all-atom molecular dynamics (MD) simulations that are widely used in the studies of biomolecules ${ }^{14,15}$ along with efficient docking simulations that are commonly applied in drug screening to investigate how rutin, a key component in many traditional antiviral medicines, interacts with the Mpro's pocket, aiming to repurpose and optimize it for the inhibition of SARS-CoV-2's main protease. Moreover, with a deeper understanding of the mechanism responsible for the rutin-Mpro recognition and binding, we were able to design and develop a more potent rutin analog for inhibiting the Mpro.

Previously, we performed MD simulations to equilibrate the crystal structure of the SARS-CoV-2 Mpro (PDB: 6LU7) in a physiologically relevant environment. ${ }^{16}$ With the equilibrated Mpro's pocket for substrate binding, we employed AutoDock Vina ${ }^{17}$ to predict the binding mode and affinity for rutin. The best pose of rutin with a binding affinity of -9.0 $\mathrm{kcal} / \mathrm{mol}$ is illustrated in Figs. 1a-b, highlighting that the entire rutin molecule was snugly harbored inside the Mpro's pocket. We then carried out the all-atom molecular dynamics simulations to study the stability of rutin inside the Mpro's pocket with a $0.15 \mathrm{M} \mathrm{KCl}$ electrolyte. The simulation system is illustrated in Fig. 1c. The detailed docking and MD simulation protocols are provided in the Methods section.

In two independent MD simulations (Sim-1 and Sim-2) of rutin in the Mpro's pocket, the Mpro's secondary structure appeared to be very stable as evidenced by the root mean square deviations (RMSDs) of the Mpro's backbone remaining at less than $2 \AA$ over the whole simulation time in both trajectories (black lines in Figs. 2a and 2b). Meanwhile, as a small molecule, the RMSDs of rutin is slightly larger or at least comparable to those of the Mpro (orange lines in Figs. 2a and 2b), indicating its flexible conformations. More importantly, if we aligned the Mpro's pocket first, the calculated RMSDs of the rutin molecule became even larger $(\sim 4.5 \AA$, blue lines in Figs. $2 \mathrm{a}$ and $2 \mathrm{~b})$, which suggests that the rutin molecule or a part of it had drifted away from its original docking pose. We note that generally this phenomenon is attributed to the less accurate account for the effect of water in docking 
studies.

Energetically, we calculated the interaction potential energies (including both van der Waals and electrostatic ones) between rutin and Mpro, as well as between rutin and the solution (including water and ions). Interestingly, from both MD simulation trajectories, we found that the potential energies between rutin and Mpro increased, suggesting that their interactions (purple lines in Figs. 2c and 2d) became weaker. On the other hand, the interaction energies between rutin and the solution showed a decrease, indicating that the rutin-water interaction became stronger. Given the fact that there are ten hydroxyl groups in one rutin molecule, it is not surprising to observe the favorable rutin-water interaction in the all-atom MD simulation. Note that in Fig. 2d (at around $200 \mathrm{~ns}$ in Sim-2), there is a temporarily dip (favorable) of the rutin-Mpro interaction energies corresponding to a briefly rise (unfavorable) of the rutin-solution interaction energies, which interestingly is due to the drifting of the C-terminal of Mpro's other monomer (without the bound rutin molecule; colored in orange in Fig. 1c) towards the rutin molecule. Remarkably, this C-terminal acted as a "lid" above the Mpro's pocket, covering the rutin molecule and thus enhancing the overall rutin-protein interaction energies. However, after about 20 ns, this C-terminal diffused away and the potential energies switched back to previous values (Fig. 2d).

Consistent with the above results, we highlight from the MD trajectories that the rutin molecule was mobile inside the Mpro's pocket and underwent conformational changes in Figs. 2e to 21 (also see movies in Supporting Information). The major difference between Sim-1 and Sim-2 lies in the position of rutin inside the Mpro's pocket after the equilibration. In Sim-1, during the first 24 ns (Figs. 2e and 2f), the entire rutin molecule shifted horizontally out of one branch of the (flipped) Y-shaped binding pocket, accompanied with rotations of the two sugar rings in the rutinose moiety. In the following $20 \mathrm{~ns}$ or so, more conformation changes occurred, e.g. the benzene ring in the quercetin moiety rotated about $90^{\circ}$ (Fig. $2 \mathrm{~g})$. At about $60 \mathrm{~ns}$, the entire molecule drifted further into the other branch of the Yshaped pocket (Fig. 2h). After that, the rutin molecule remained at this location for the 

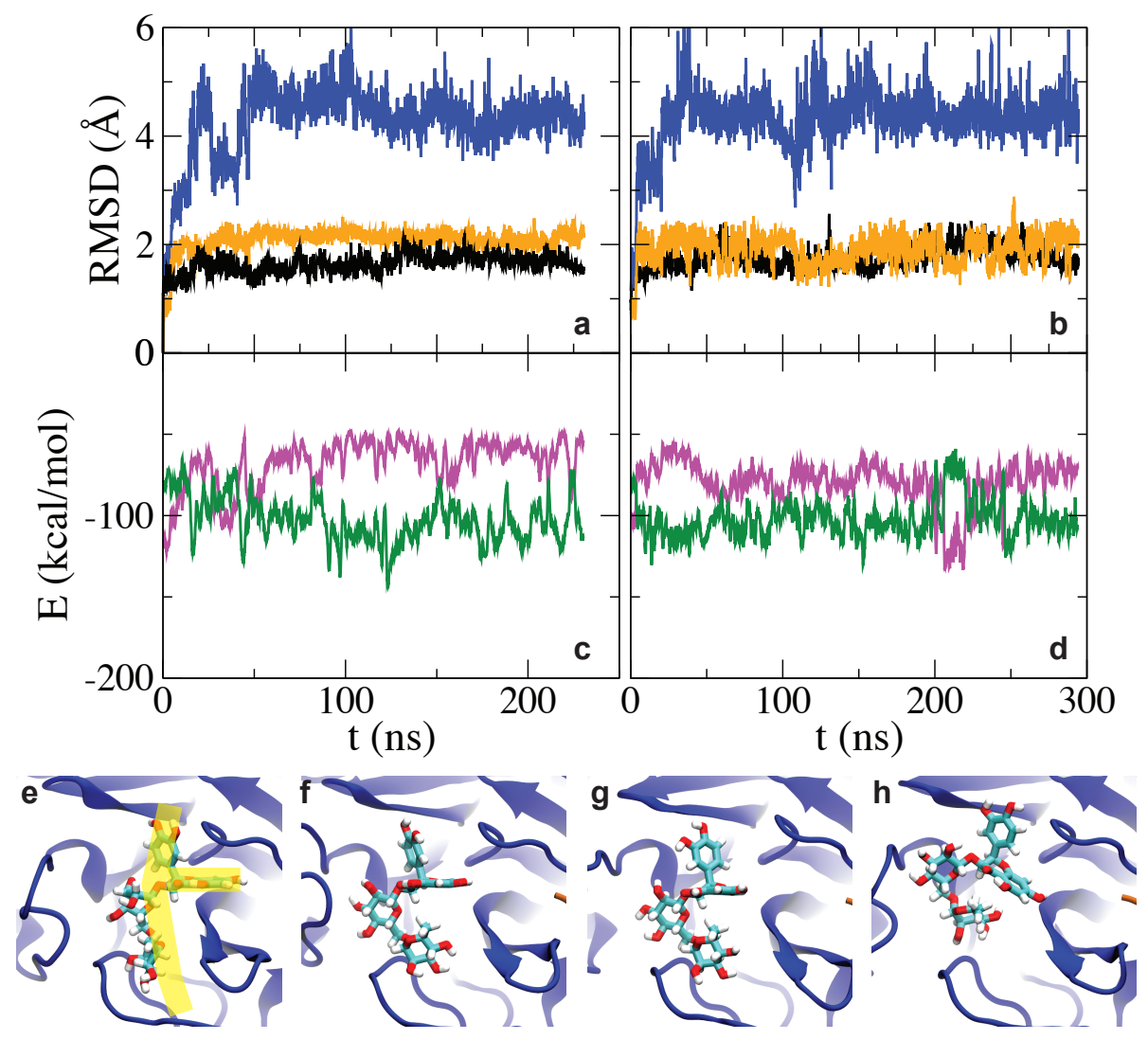

Sim-1: 0 ns

$24 \mathrm{~ns}$

$45 \mathrm{~ns}$

$60 \mathrm{~ns}$

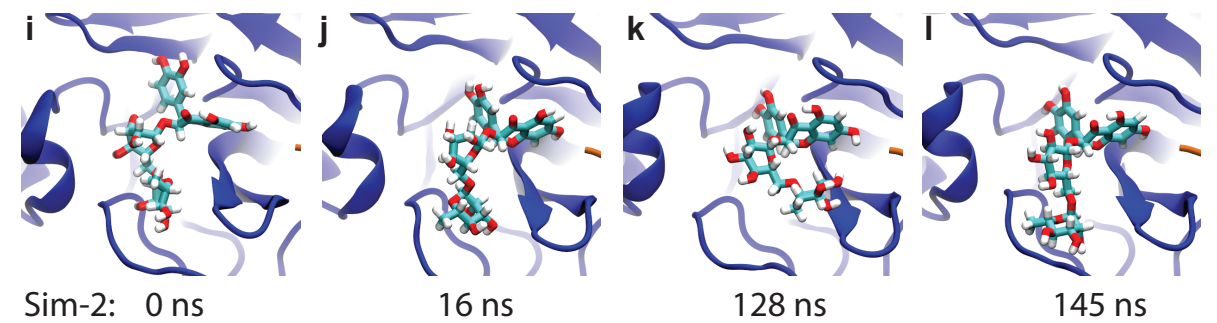

Figure 2: Two independent MD simulations of rutin bound inside the Mpro's pocket. a,b) The time-dependent root mean square deviations (RMSDs) for the protein backbone (black lines), for the bound rutin molecule calculated against the best pose from the docking study (orange lines), and for the rutin molecule after the protein alignment (blue lines). c, d) Potential energies between rutin and the Mpro (purple), and between rutin and the solution (green). Results from Sim-1 are shown in (a) and (c), while results from Sim-2 are shown in (b) and (d). e-h) Illustration of rutin's conformations inside the Mpro's pocket, at $t=0$, 24, 45 and 60 ns (Sim-1), respectively. i-l) Illustration of rutin's conformations inside the Mpro's pocket, at $t=0,16,128$ and $145 \mathrm{~ns}$ (Sim-2), respectively. 
rest of about $170 \mathrm{~ns}$ of simulation. It is worth mentioning that the rutin molecule is right above the Mpro's active site (including Ser46 and Cys145) as shown in Fig. S1a (Supporting Information) and thus can inhibit Mpro.

In Sim-2, the quercetin moiety stably occupied the two branches of the Y-shaped pocket, with slight conformational change (Figs. 2i to 2l). Besides the hydrophobic interaction between the benzene rings in the quercetin moiety and hydrophobic residues (such as Met164) in Mpro, the hydroxyl groups on the quercetin moiety also formed stable hydrogen bonds with residues His41 and His163 (see Fig. S1b in Supporting Information). Initially, the rutinose moiety occupied the so called "anchor site" of the binding pocket, that is usually filled by a hydrophobic group of a bound ligand to enhance the binding affinity. ${ }^{16}$ Due to the hydrophilic interaction with water, most hydroxyl groups on the two sugar rings of the rutinose moiety faced toward water, after the rotational adjustment of the rutinose moiety (around 16 ns, Fig. 2j). Besides this, the whole rutinose moiety moved further into water and stayed above the binding pocket. Consequently, it can swing widely (not sterically confined by the pocket anymore), as shown in Figs. 2k and 2l. Despite the dramatic changes in the rutinose moiety, the quercetin part was firmly bound inside the pocket for the entire simulation time $(\sim 300$ ns). Overall, the rutin molecule was stably bound to the Mpro's pocket and can sterically block the substrate binding (See Fig. S1b in Supporting Information).

Given the nearly perfect fitting of the rutin's structure in the Mpro's pocket from the docking study (Figs. 1a and 1b) and the obvious drawbacks of the rutin's binding to the Mpro's pocket as revealed in Sim-2, we were motivated to design a new drug molecule based on rutin (Fig. 3a). As demonstrated in experiment previously, a hydrophobic moiety inside the anchor site can help stabilize/enhance the ligand binding. ${ }^{12,18,19}$ In the designed drug molecule, we replaced two hydroxyl groups in each sugar ring with two methyl groups respectively (Fig. 3b). To satisfy Lipinski's rule of five, ${ }^{20}$ we further replaced one hydroxyl group in the benzene ring with a hydrogen atom (Fig. 3b). As a result, the new molecule (referred as rutin-M hereafter) is much more hydrophobic than rutin. 
a

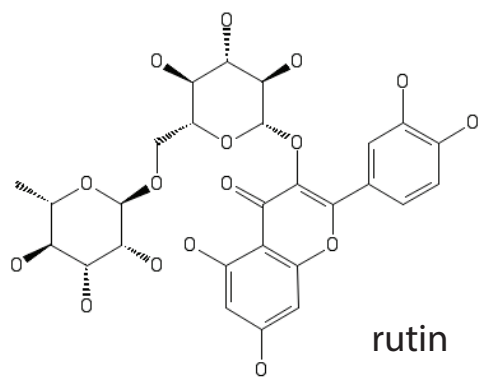

b

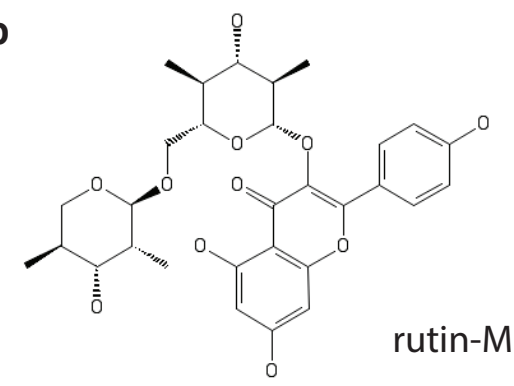

C
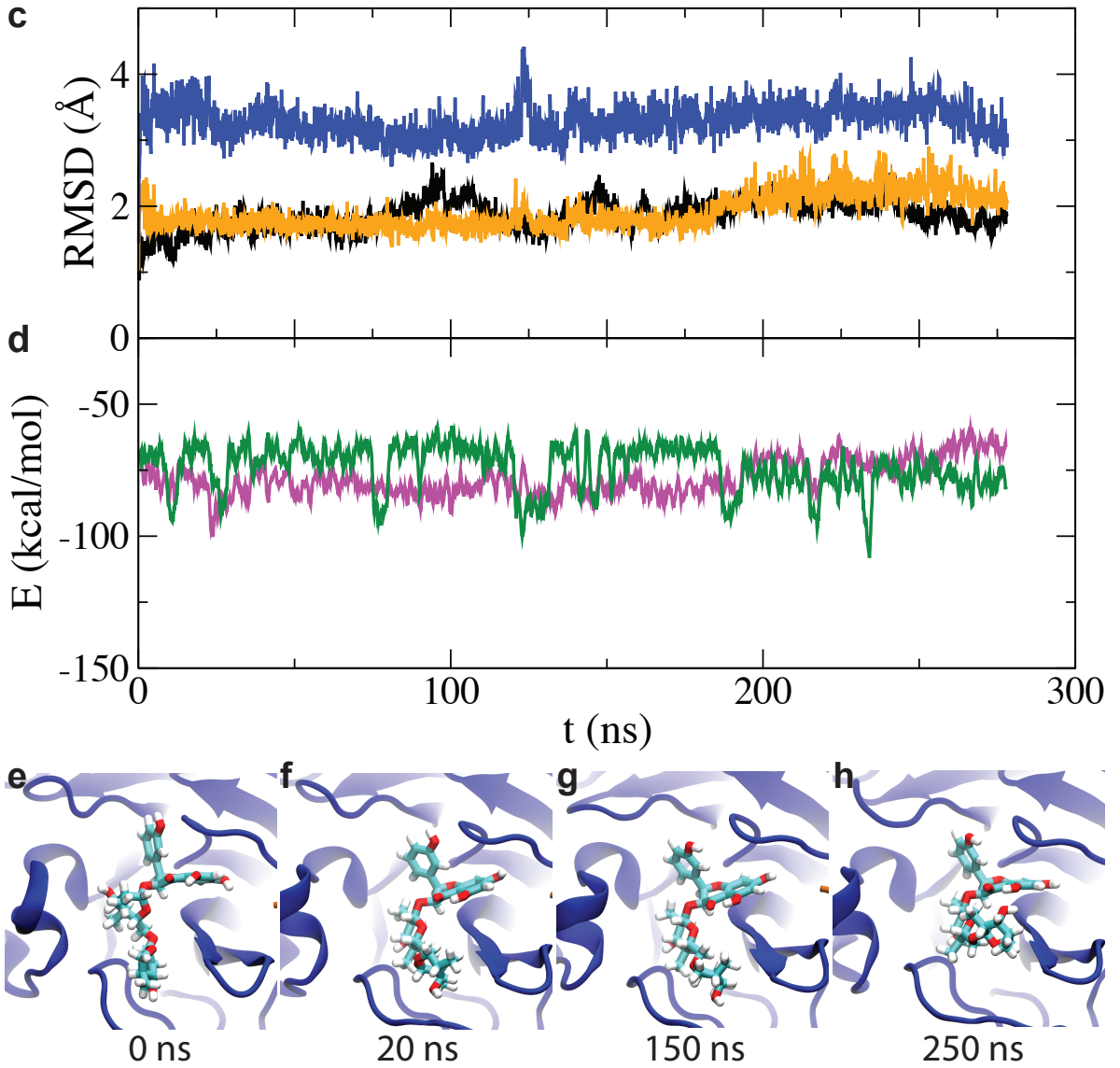

Figure 3: MD simulation of rutin-M bound inside the Mpro's pocket. a,b) The comparison between structures of rutin and rutin-M. c) Time-dependent root mean square deviations (RMSDs) for the protein backbone (black); time-dependent RMSDs for the bound rutin-M, calculated against the best pose in docking, after the ligand alignment (orange) and the protein alignment (blue). d) Potential energies between rutin-M and the Mpro (purple), and between rutin-M and the solution (green). e-h) Top views of conformations of rutin-M inside the Mpro's pocket, at $t=0,20,150$ and $250 \mathrm{~ns}$, respectively. 
Using the same simulation protocol, we carried out MD simulation to investigate the binding stability of rutin-M. The calculated RMSDs for Mpro and rutin-M are comparable to those obtained from Sim-1 and Sim-2 for the rutin-Mpro complex (Fig. 3c). However, with the Mpro's backbone (and thus the Mpro's pocket) aligned, the RMSDs of rutin-M is only about $3.3 \AA$, which is significantly smaller than the value $(\sim 4.5 \AA)$ for rutin (Figs. $2 \mathrm{a}$ and $2 \mathrm{~b}$ ). These RMSD results suggest that rutin-M is as flexible as rutin but is more stable inside the Mpro's pocket. Energetically, the average interaction energy between rutin-M and Mpro is about -75 kcal/mol (Fig. 3d, purple line), which is stronger than that ( $\sim$ $70 \mathrm{kcal} / \mathrm{mol}$ ) between rutin and Mpro in Sim-2 (Fig. 2d, purple line), resulting from the increased hydrophobic interaction between rutin-M and Mpro. It is worth mentioning that the docking result for rutin-M is about $-8.8 \mathrm{kcal} / \mathrm{mol}$, that is comparable to the one for rutin. Notably, the interaction energies between rutin-M and the solution are around $-70 \mathrm{kcal} / \mathrm{mol}$ (Fig. 3d, green line) that is much larger than $\sim-105 \mathrm{kcal} / \mathrm{mol}$ for rutin in Sim-2 (Fig. 2d, green line). The weaker interaction between rutin-M and water/ions is consistent with the design of rutin-M shown in Fig. 3b.

From the simulation trajectory, we found that the modified sugar ring (first) connected to the quercetin moiety sank deeply into the bottom of the Mpro's pocket, due to the strong hydrophobic interaction between two added methyl groups in rutin-M and the hydrophobic residues (such as Met49 and Met165) in Mpro (Figs. 3f to 3h). Thus, the modifications on this ring has proven fruitful and enhanced the binding between rutin-M and the Mpro's pocket. The other modified sugar ring (second) stayed in the end of the Y-shaped pocket for almost $200 \mathrm{~ns}$ (Figs. 3f and 3g). However, induced by thermal fluctuation, the temporal widening of the Mpro's pocket eventually allowed the second ring to escape the pocket (Fig. 3h). Consistently, as shown in Fig. 3d, the interaction potential energies between rutin$\mathrm{M}$ and Mpro increased (less favorable) after about $200 \mathrm{~ns}$ while the ones between rutin-M and the solution decreased (more favorable) accordingly. We note that, during the entire simulation time of about $275 \mathrm{~ns}$, the first ring and the quercetin moiety were stably bound 
inside the Mpro's pocket.

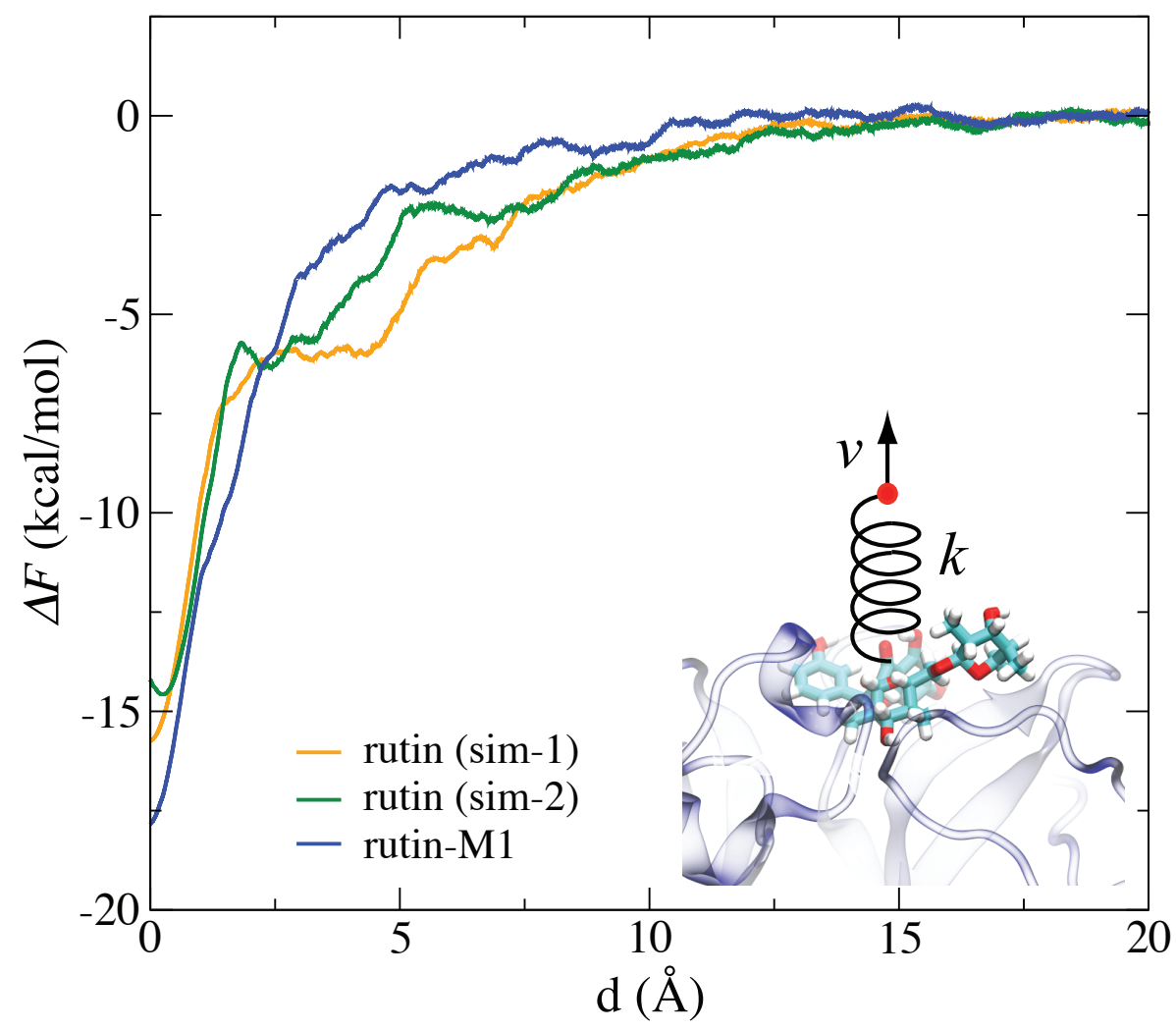

Figure 4: Binding free energy differences for rutins' entry into water from the Mpro's pocket. Inset: the system set-up for the steered molecular dynamics simulation and the side view of rutin-M in the Mpro's pocket.

To estimate the binding free energies of rutin and rutin-M inside the Mpro's pocket, we applied the steered molecular dynamics (SMD) simulation to pull the drug molecules out of their respective binding sites using a harmonic spring. As illustrated in Fig. 4, one end of the spring was attached to the center of mass of the drug molecule and the other end of the spring was attached to a pulling stage that moved at a constant velocity $(1 \AA / \mathrm{ns})$. More details are provided in the Methods section.

Totally, ten independent SMD simulations were carried out using a stiff harmonic spring ${ }^{21}$ $(k=2 \mathrm{nN} / \AA)$ to pull each drug molecule perpendicularly out of the Mpro's pocket. The distance-dependent works $W$ are shown in Fig. S2 in Supporting Information. From these simulations, we used Jazynski equality, ${ }^{22} e^{\Delta F / k T}=\left\langle e^{-W / k T}>\right.$, to calculate the binding free 
energy difference $\Delta F$ (or the potential of mean force) for the drug-Mpro binding, where $k$ is the Boltzmann constant and $T$ is the temperature. Figure 4 shows the free energy differences for the two rutins (in Sim-1 and Sim-2) and rutin-M moving from their respective binding sites to the solution. From this plot, we conclude that the estimated binding free energies for the rutins in Sim-1 and Sim-2 are comparable with the former being $-15.7 \mathrm{kcal} / \mathrm{mol}$ and later being $-14.6 \mathrm{kcal} / \mathrm{mol}$. In contrast, the estimated binding free energy for rutin$\mathrm{M}$ is $-17.0 \mathrm{kcal} / \mathrm{mol}$, which confirms that the suggested modification (shown in Fig. 3b) overall enhanced the binding affinity of rutin-M inside the Mpro's pocket. This significant improvement is mainly due to the modified first-sugar ring that lied deeply inside the binding pocket. Note that in the unmodified rutin the first sugar ring along with the second one are both outside the Mpro's pocket (or in water, Sim-2).

In this study, rutin and rutin-M have the docking scores of $-9.0 \mathrm{kcal} / \mathrm{mol}$ and $-8.8 \mathrm{kcal} / \mathrm{mol}$ respectively to Mpro, out-performing a mechanism-based Mpro inhibitor N3, ${ }^{16}$ which was optimized for targeting viral main proteases such as SARS-CoV Mpro. The Y-shaped scaffold of the bound rutin molecule fits snugly into the Mpro's pocket with many medicinal and chemical improvement potentials. We envision flavonoid scaffold represented by rutin as a new versatile backbone for designing specific reversible and irreversible viral protease inhibitors (drug) outside of the chemical spaces defined by the N3 series, which mimic the viral peptide substrates.

Viruses have a busy life to live, especially for RNA virus like SARS-CoV-2 which proliferates at a much higher speed than most other species. Viral proteins need to be made and mature by Mpro in large quantities to meet the speed of viral proliferation. In consequence, Mpro would have to adopt a high turn-over rate of substrate. Thus the kinetics of a small molecule inhibitor binding with Mpro $\left(K_{\text {on }}\right.$ and $\left.K_{\text {off }}\right)$ need to be particularly well-tuned to compete with viral protein peptide substrates. Non-binding molecule such as Cinanserin (despite a relatively good static docking score, $-6.2 \mathrm{kcal} / \mathrm{mol}^{16}$ ) would have difficulties in inhibiting the Mpro sufficiently to hamper the virus proliferation. A better understanding 
of the dynamical inhibitor/Mpro interaction is required to design the efficacious inhibitor. In this case, covalent modifier of Mpro would stand a much better chance to provide the inhibition necessary for viral inhibition.

As a natural product, rutin wasn't optimized for binding with viral protease nor its absorption, distribution, metabolism, and excretion (ADME) properties being perfect for an orally available drug, which leaves a lot of room for improvement. During the molecular modeling, we also found the 4' and 5' position hydroxyl-groups in eastern moiety (Fig. 3a) are in close proximity with the catalytic center of Cys145 (4.4 $\AA$ away) and an adjacent Ser46 for sufficient long time (Fig. S1 in Supporting Information). This proximity provides unique opportunities for further designing a new rutin-based SARS-CoV-2 Mpro covalent inhibitors with the addition of moieties like $\alpha$-ketoamide, sulfonyl fluoride groups. It is tempting to postulate that in the case of irreversible inhibitors, the inhibitor $K_{\text {off }}$ would be infinity until the end of the proteins' natural turn-over rate, allowing a much smaller dose to achieve the same effects. To avoid the peripheral toxicity that is pertinent to many irreversible inhibitors, it is also advisable to nebulize the drug with inhalable solution and/or apply it locally in salivary glands and pharynx.

Rutin is soluble in water $(125 \mathrm{mg} / \mathrm{L})$, however, the metabolism of the pure form in the guts and liver is almost complete, making the parental molecule bioavailability almost zero. However, rutin in vegetable and herbal medicines seems to have better bioavailability evidenced by the mass spectrum. ${ }^{11}$ It is likely that the recent discovered plant nanometer scale lipid microvesicles (or plant exosomes) could have helped to carry rutin into the body, preventing it from metabolism and increasing its bioavailability.

Since SARS-CoV-2 proliferates in large quantities in salivary glands and pharynx during the asymptomatic phase, patients end up spreading out extremely contagious droplets containing the virus, subsequently infecting the lower respiratory system. It is thus beneficial to drink blended herbal juice containing natural rutin (buckwheat, unpeeled apples, asparagus, elderflower, sophora flower-bud etc..) and rinse mouth and pharynx with ex- 
tended time to reduce the viral load. This approach could provide sufficient access of rutin and other anti-viral flavonoids to the infected cells without the liability of dilution and low bioavailability.

In conclusion, we investigated in silico how rutin, a cheap and widely available natural product in vegetable/fruits/herbal medicines, could be modified as a potential inhibitor for SARS-CoV-2 Mpro. Guided by the docking study, we discovered that rutin can fit inside the Mpro's binding pocket. From MD simulations, we further investigated the rutin's binding stability and revealed that the rutinose moiety is too hydrophilic to remain inside the Mpro's pocket although the quercertin moiety was stably bound. We then designed a more hydrophobic analog of rutin (rutin-M) and our results show that one part of the modified rutinose moiety stably stayed inside the Mpro pocket and the revealed binding affinity was higher for rutin-M than for rutin. Based on our studies, we postulated two possible approaches to design rutin analogs: (1) optimizing chemical groups on rutin to enhance the binding affinity and improve the bioavailabilty; (2) utilizing the designed rutin analogs as candidates for irreversible binding. Additionally, we discussed possible avenues on how to deliver rutin and its analogs to inflamed organs for potential treatment of COVID19. We expect that our in silico studies could facilitate/accelerate the future structure-based design of high-potent drug molecules targeting the Mpro of SARS-CoV-2.

\section{Methods}

Molecular dynamics simulation The MD simulations were carried out on an IBM Power-cluster, using the $\mathrm{NAMD}^{23}$ package. In Fig. 1, the dimer structure of the Mpro (colored in blue and orange respectively) is in the cartoon representation while the rutin molecule is in the van der Waals sphere representation. The complex was then solvated in a water box with a dimension of $97.4 \times 97.4 \times 97.4 \quad \AA^{3}$. The $\mathrm{KCl}$ electrolyte concentration was $0.15 \mathrm{M}$ (including $80 \mathrm{~K}^{+}$and $80 \mathrm{Cl}^{-}$ions). Extra $8 \mathrm{~K}^{+}$ions were added to neutralize 
the Mpro's charge. We applied the TIP3P model ${ }^{24,25}$ for water, standard ion force field ${ }^{26}$ and the CHARMM36 force field ${ }^{27}$ for the protein. The force field parameters for rutin were obtained from SwissParam. ${ }^{28}$ A smooth cutoff (10-12 A) was used to compute van der Waals potential energies. Electrostatic interactions were calculated using the particle-mesh Ewald (PME) method (grid size $\sim 1 \AA$ ). With the SETTLE algorithm ${ }^{29}$ enabled to keep all bonds rigid, the simulation time-step was 2 fs

After the minimization, the entire simulated system was first equilibrated for 1 ns at 1 bar and $300 \mathrm{~K}$, with all backbone atoms in the Mpro and non-hydrogen atoms in the rutin molecule harmonically restrained (spring constant $k=1 \mathrm{kcal} / \mathrm{mol} / \AA^{2}$ ). In the subsequent production simulation, the restraint was removed and the Mpro was further equilibrated in the NPT ensemble for about $230 \mathrm{~ns}$. The Langevin dynamics was applied to maintain the constant temperature $(300 \mathrm{~K})$ in the simulated system and the pressure was kept constant at 1 bar using the Nosé-Hoover method. ${ }^{30}$

Steered molecular dynamics simulation To estimate the binding free energies of rutin and its modified analog, we used the steered molecular dynamics simulations to calculate potentials of mean force. The spring constant was $2 \mathrm{nN} / \AA$ and the pulling velocity was 1 $\AA /$ ns. The center of mass of each ligand molecule was pulled perpendicularly out of the Mpro's binding pocket, while the backbone of the Mpro was harmonically $\left(5 \mathrm{kcal} / \mathrm{mol} / \AA^{2}\right)$ restrained to its original position.

Docking We carried out the docking calculations on an IBM power node with 24 physical cores and 192 CPUs. For preparation of the ligands and target protein to be applied in AutoDock Vina, we used the scripts prepare_ligand4.py and prepare_receptor4.py provided with the AutoDock Tools ${ }^{17,31}$ suite to generate the corresponding input files in PDBQT format which extends the PDB format with additional fields of partial charge and atom type. In order to increase the chance of finding the minimum binding energy to predict where and how a putative ligand can best bind the target protein, we set the exhaustiveness 
parameter of the program to 100 for each search.

\section{Competing Interests}

T. H., H. W., W. C. and B. L. declare no conflicts of interest.

\section{Acknowledgement}

B. L. gratefully acknowledges the computing resource and the financial support from the IBM Bluegene Science Program.

\section{Supporting Information Available}

The movies from the trajectories of Sim-1 and Sim-2, showing dynamic binding of rutin inside the Mpro's pocket; Fig.S1 showing rutin-Mpro binding in Sim-1 and Sim-2; Fig.S2 showing the data from SMD simulations.

\section{References}

1. Kuiken, T.; Fouchier, R. A.; Schutten, M.; Rimmelzwaan, G. F.; Van Amerongen, G.; van Riel, D.; Laman, J. D.; de Jong, T.; van Doornum, G.; Lim, W. et al. Newly discovered coronavirus as the primary cause of severe acute respiratory syndrome. The Lancet 2003, 362, 263-270.

2. Zaki, A. M.; Van Boheemen, S.; Bestebroer, T. M.; Osterhaus, A. D.; Fouchier, R. A. Isolation of a novel coronavirus from a man with pneumonia in Saudi Arabia. New England Journal of Medicine 2012, 367, 1814-1820.

3. Li, M.; Wang, J. Prospect of ambroxol in the treatment of COVID-2019. The Chinese Journal of Clinical Pharmacology 2020, 
4. Jo, S.; Kim, S.; Shin, D. H.; Kim, M.-S. Inhibition of SARS-CoV 3CL protease by flavonoids. Journal of enzyme inhibition and medicinal chemistry 2020, 35, 145-151.

5. Yao, C.; Xi, C.; Hu, K.; Gao, W.; Cai, X.; Qin, J.; Lv, S.; Du, C.; Wei, Y. Inhibition of enterovirus 71 replication and viral 3C protease by quercetin. Virology Journal 2018, $15,116$.

6. Chéron, N.; Yu, C.; Kolawole, A. O.; Shakhnovich, E. I.; Wobus, C. E. Repurposing of rutin for the inhibition of norovirus replication. Archives of Virology 2015, 160, 23532358.

7. Choi, J.-H.; Kim, D.-W.; Park, S.-E.; Lee, H.-J.; Kim, K.-M.; Kim, K.-J.; Kim, M.-K.; Kim, S.-J.; Kim, S. Anti-thrombotic effect of rutin isolated from Dendropanax morbifera Leveille. Journal of Bioscience and Bioengineering 2015, 120, 181-186.

8. Ganeshpurkar, A.; Saluja, A. K. The pharmacological potential of rutin. Saudi Pharm. J. 2017, 25, 149-164.

9. Zhang, Y.; Xiao, M.; Zhang, S.; Xia, P.; Cao, W.; Jiang, W.; Chen, H.; Ding, X.; Zhao, H.; Zhang, H. et al. Coagulopathy and antiphospholipid antibodies in patients with Covid-19. New England Journal of Medicine 2020,

10. Runfeng, L.; Yunlong, H.; Jicheng, H.; Weiqi, P.; Qinhai, M.; Yongxia, S.; Chufang, L.; Jin, Z.; Zhenhua, J.; Haiming, J. et al. Lianhuaqingwen exerts anti-viral and antiinflammatory activity against novel coronavirus (SARS-CoV-2). Pharmacological Research 2020, 104761.

11. Hua, Y.; Weina, J.; Jinping, L.; Yan, Z.; Chun-hua, W. Analysis of absorbed components in rat plasma after oral administration of Lianhua Qingwen capsules by UPLC-Q-TOFMS. Tianjin Journal of Traditional Chinese Medicine 2016, 33, 756. 
12. Jin, Z.; Du, X.; Xu, Y.; Deng, Y.; Liu, M.; Zhao, Y.; Zhang, B.; Li, X.; Zhang, L.; Peng, C. et al. Structure of Mpro from COVID-19 virus and discovery of its inhibitors. Nature 2020,

13. Si, L.; Bai, H.; Rodas, M.; Cao, W.; Oh, C. Y.; Jiang, A.; Nurani, A.; Zhu, D. Y.; Goyal, G.; Gilpin, S. et al. Human organs-on-chips as tools for repurposing approved drugs as potential influenza and COVID19 therapeutics in viral pandemics. bioRxiv 2020 ,

14. Karplus, M.; McCammon, J. Molecular dynamics simulations of biomolecules. Nature Struct. Biol. 2002, 265, 654-652.

15. Luan, B.; Xu, G.; Feng, M.; Cong, L.; Zhou, R. Combined Computational-Experimental Approach to Explore the Molecular Mechanism of SaCas9 with a Broadened DNA Targeting Range. J. Am. Chem. Soc. 2019, 141, 6545-6552.

16. Huynh, T.; Wang, H.; Luan, B. in silico Exploration of Molecular Mechanism and Potency Ranking of Clinically Oriented Drugs for Inhibiting SARS-CoV-2's Main Protease. ChemRxiv 2020,

17. Trott, O.; Olson, A. J. AutoDock Vina: improving the speed and accuracy of docking with a new scoring function, efficient optimization, and multithreading. J. Comput. Chem. 2010, 31, 455-461.

18. Dai, W.; Zhang, B.; Su, H.; Li, J.; Zhao, Y.; Xie, X.; Jin, Z.; Liu, F.; Li, C.; Li, Y. et al. Structure-based design of antiviral drug candidates targeting the SARS-CoV-2 main protease. Science $\mathbf{2 0 2 0}$,

19. Zhang, L.; Lin, D.; Sun, X.; Curth, U.; Drosten, C.; Sauerhering, L.; Becker, S.; Rox, K.; Hilgenfeld, R. Crystal structure of SARS-CoV-2 main protease provides a basis for design of improved $\alpha$-ketoamide inhibitors. Science 2020, 
20. Lipinski, C. A.; Lombardo, F.; Dominy, B. W.; Feeney, P. J. Experimental and computational approaches to estimate solubility and permeability in drug discovery and development settings. Advanced drug delivery reviews 1997, 23, 3-25.

21. Park, S.; Schulten, K. Calculating potentials of mean force from steered molecular dynamics simulations. J. Chem. Phys. 2004, 120, 5946-5961.

22. Jarzynski, C. Nonequilibrium Equality for Free Energy Differences. Phys. Rev. Lett. 1997, 78, 2690-2693.

23. Phillips, et.al., J. C. J. Comp. Chem. 2005, 26, 1781.

24. Jorgensen, W. L.; Chandrasekhar, J.; Madura, J. D.; Impey, R. W.; Klein, M. L. Comparison of Simple Potential Functions for Simulating Liquid Water. J. Chem. Phys. 1983, 79, 926-935.

25. Neria, E.; Fischer, S.; Karplus, M. Simulation of Activation Free Energies in Molecular Systems. J. Chem. Phys. 1996, 105, 1902-1921.

26. Beglov, D.; Roux, B. Finite representation of an infinite bulk system: Solvent boundary potential for computer simulations. J. Chem. Phys. 1994, 100, 9050-9063.

27. Huang, J.; MacKerell Jr, A. D. CHARMM36 all-atom additive protein force field: Validation based on comparison to NMR data. J. comput. Chem. 2013, 34, 2135-2145.

28. Zoete, V.; Cuendet, M. A.; Grosdidier, A.; Michielin, O. SwissParam: a fast force field generation tool for small organic molecules. J. Comput. Chem. 2011, 32, 2359-2368.

29. Miyamoto, S.; Kollman, P. A. SETTLE: An Analytical Version of the SHAKE and RATTLE Algorithm for Rigid Water Molecules. J. Comp. Chem. 1992, 13, 952-962.

30. Martinetz, T.; Schulten, K. Topology Representing Networks. Neur. Netw. 1994, 7, 507-522. 
31. Morris, G. M.; Huey, R.; Lindstrom, W.; Sanner, M. F.; Belew, R. K.; Goodsell, D. S.; Olson, A. J. AutoDock4 and AutoDockTools4: Automated docking with selective receptor flexibility. J. Comput. Chem. 2009, 30, 2785-2791. 